\title{
Switching Circuits Simplifications Using Binary Coded Octal Minterms
}

\author{
Sahadev Roy
}

\begin{abstract}
In this paper, a simple approach for detection of adjacent minterms and minimization technique is proposed. The proposed method factorized each minterms and 'don't-care' conditions into a group of maximum three inputs variables and these groups of minterms further coupled as per laws of simplification. The entire detection procedure is described here by mix binary-octal representations. A chart proposed in this paper for easy detection of adjacent minterms with fewer numbers of comparisons and can use for any number of variables. Logic adjacency property is played a significant role for logic circuit simplification. This novel method reduces the complexity of two-level simplification procedure. This paper also represents the performance of octal minterms based combinational circuit minimization technique in comparison with other traditional methods. The paper deals with a method based on representing minterms in the octal system that can be better suited for the manual method of logic minimization and hence may be worth considering.
\end{abstract}

Keywords

Algorithm for digital circuit simplification; Boolean function simplification; Combinational logic minimization; Binary coded octal minterms; Octal minterms

\section{INTRODUCTION}

Adjacent minterms identifications and manipulations are one of the essential tasks for designing of automatic control systems, designing of FPGA-based systems and for designing of many other digital circuits [1]. In general, most of the gatelevel minimization procedures are based on the absorptions of the adjoining minterms. Any combinational circuit minimization techniques are classified based on adjacent minterms detection methodologies (i) exact methods, which are complex and required considerable processing time but provide the least number of sum-of-products terms and (ii) heuristic methods, provide near optimum solutions within a reasonable time [2, 3]. In 1936, Claude E. Shannon initiated the use of Boolean algebra in the analysis and design of switching circuits [4]. He also identifies a set of formulas, which are very useful for detection of adjacent minterms pair and digital circuit minimization techniques. In 1953, an inspection based method proposed by Veitch [5] which was modified by Karnaugh [6]. The Quine-McCluskey algorithm is the first computerized grouping of minterms based minimization algorithm $[7,8]$. Other minimization techniques like MINI [9], Boolean Minimizer BOOM [10] algorithms can handle a large number of the input variable and based on heuristic detection method. The major drawback of these algorithms is the limited size of the problems due to huge memory space is required. For a large number of input variables such as in Built-in-Self-Test (BIST) [11, 12], System-on-a-chip (SoC) designs or VLSI system design [13, 14], neuron-like operation (sum of product) [15] required minimization times are extremely long. Here, an alternate way of complexity minimization is suggested that those input conditions for that output become logic high or in don't care state in a truth table easily converted into an equivalent octal number. The paper proposed an efficient and fast minimization process using octal code representation. This technique is also useful for minimization of multiple input multiple output digital circuits.

Any logic function that can be realized in various forms and one of these proves to be the best suitable for a particular application under certain design consideration. Any switching functions having more than two variables can be expressed by using three fundamental logical relations complements, ANDing, and Oring. Any switching activity also can be represented either ANDing or Oring of all variables where each variable appears once either in an active state (1) or inactive state (0). Serrell introduced minterms (m) as any one of the $2^{n}$ distinct intersections (Oring) and maxterms (M) as any one of the $2^{n}$ distinct union (ANDing) of all $\mathrm{n}$ input variables [16]. In the both cases, only one appearance of any variable is assigned in one of the terms and has two allowable conditions either in an active condition (1) or inactive condition (0) but not the both, which is popularly known as literals. So, the minterms are the ANDing (logical product) maxterms are the Oring (logical sum) of all the literals with or without complement implicated in a logic circuit. It is possible to represent any Boolean expression entirely by using either minterms or maxterms are known as the canonical Sum-ofProducts (SOP) or Product-of-Sums (POS) respectively. Any SOP or POS expressions which are represented by literals are unsuitable for both write and synthesis. For convenience, an

Authors Details:

* Sahadev Roy

Dept. of ECE, National Institute of Technology, Arunachal Pradesh, India

e-mail:sdr.ece@nitap.in

Subject Category: Engineering

Sub Category: Digital VLSI 
alternate shorthand notation is used for describing any switching functions. Functions are realized by literals maintaining a proper sequence, and the successive literals are to be interpreted as the coefficients of consecutive integral powers of 2 (binary radix) and finally represented as Decimal Coded Minterms (DCm) or Decimal Coded Maxterms (DCM). However, for a large number of input variables, this type of coding technique is required an enormous calculation. For an illustration, we consider a seven input switching systems, and its switching conditions are tabulated in Table 1.

Table 1. Illustration of proposed octal truth table

\begin{tabular}{|c|c|c|c|c|c|c|c|c|}
\hline \multicolumn{7}{|c|}{ Variables } & \multirow{3}{*}{$\begin{array}{c}\text { Proposed } \\
\text { Scheme } \\
\text { Octal } \\
\text { Minterms }\end{array}$} & \multirow{3}{*}{$\begin{array}{c}\text { Traditional } \\
\text { Scheme } \\
\text { Decimal } \\
\text { Minterms }\end{array}$} \\
\hline G2 & \multicolumn{3}{|c|}{ G1 } & \multicolumn{3}{|c|}{ G0 } & & \\
\hline A & B & $\mathrm{C}$ & D & E & $\mathrm{F}$ & G & & \\
\hline 0 & 0 & 0 & 0 & 0 & 1 & 0 & 002 & 2 \\
\hline 0 & 0 & 0 & 1 & 0 & 1 & 0 & 012 & 10 \\
\hline 0 & 0 & 0 & 1 & 0 & 1 & 1 & 013 & 11 \\
\hline 0 & 0 & 0 & 1 & 1 & 1 & 0 & 016 & 14 \\
\hline 0 & 0 & 1 & 0 & 0 & 1 & 0 & 022 & 18 \\
\hline 0 & 0 & 1 & 1 & 0 & 1 & 0 & 032 & 26 \\
\hline 0 & 0 & 1 & 1 & 0 & 1 & 1 & 033 & 27 \\
\hline 0 & 0 & 1 & 1 & 1 & 1 & 0 & 036 & 30 \\
\hline 0 & 1 & 0 & 0 & 0 & 1 & 0 & 042 & 34 \\
\hline 0 & 1 & 0 & 1 & 0 & 1 & 1 & 053 & 43 \\
\hline 0 & 1 & 1 & 1 & 0 & 1 & 1 & 073 & 59 \\
\hline 1 & 0 & 0 & 1 & 0 & 1 & 0 & 112 & 74 \\
\hline 1 & 0 & 0 & 1 & 1 & 1 & 0 & 116 & 78 \\
\hline 1 & 0 & 1 & 1 & 0 & 1 & 0 & 132 & 90 \\
\hline 1 & 0 & 1 & 1 & 1 & 1 & 0 & 136 & 94 \\
\hline 1 & 1 & 0 & 1 & 1 & 1 & 0 & 156 & 110 \\
\hline
\end{tabular}

To obtain equivalent $\mathrm{DC} \mathrm{m}$ for each input combination the following operations required to perform,

$$
\mathrm{DCm}=64 \cdot \mathrm{a}+32 \cdot \mathrm{b}+16 \cdot \mathrm{c}+8 \cdot \mathrm{d}+4 \cdot \mathrm{e}+2 \cdot \mathrm{f}+\mathrm{g}
$$

Where, $a, b, \cdots, g$ is either 1 for logic high or 0 for logic low condition corresponding the inputs $A, B, \cdots, G$ respectively. In this illustration, for each minterms six addition and six multiplications, i.e. twelve arithmetic operations are required for each minterms. In general, for realizing $\mathrm{DCm}$ from any $\mathrm{n}$-input truth table $2^{n-1}$ arithmetic operations is required. Equation (2) is the equivalent representation using traditional shorthand notation.

$$
\begin{aligned}
f(A, B, C, D, E, F, G) \\
\qquad \sum \mathrm{m}\left(\begin{array}{c}
2,10,11,14,18,26,27,30 \\
34,43,59,74,78,90,94,110
\end{array}\right)
\end{aligned}
$$

For more convenience, an alternate shorthand notation is proposed here by using the Binary Coded Octal numbering (BCO) scheme. Pacifically, the octal number system is used to realize the bit patterns of the truth table. As per binary to octal conversion rules, initially partition into groups of three bits starting from least significant bit assigned in the truth table and each group is denoted here as G0, G1, etc. Each group consists of maximum three input variables. For this purpose, an octal truth table is proposed here as shown in Table 1 which is an alternate representation technique of truth conditions. Minterms or maxterms those are represented using BCO scheme we called 'Octal Coded Minterms' (Om) or 'Octal Coded Maxterms' (OM). The equivalent representation of Eq. (2) in this scheme is shown in Eq. (3). Suffix 8 is used to indicate minterms are octal minterms.

$$
\begin{aligned}
& f(A, B, C, D, E, F, G) \\
& =\sum \mathrm{m}_{8}\left(\begin{array}{c}
002,012,013,016,022,032,033,036 \\
042,053,073,112,116,132,136,156
\end{array}\right)
\end{aligned}
$$

The number of possible group (Gr) any $n$ input system is given by,

$$
\mathrm{Gr}=\operatorname{ceil}(\mathrm{n} / 3)
$$

Function 'ceil' used in Eq. (4) for rounds to the next larger integer when $\mathrm{n}$ is not divisible by 3 . To obtain equivalent $\mathrm{Om}$ for each input combination of the Table 1 following operations required to perform,

$$
\mathrm{Om}=(\mathrm{a})(4 \cdot \mathrm{b}+2 \cdot \mathrm{c}+\mathrm{d})(4 \cdot \mathrm{e}+2 \cdot \mathrm{f}+\mathrm{g})
$$

To realized equivalent octal code for each group number of operation required to perform,

Total no of operations

$$
= \begin{cases}4 & \text { for case } 1 \\ 2 & \text { for case } 2 \\ 0 & \text { for case } 3\end{cases}
$$

Case 1: Two additions and two multiplications operations are required to perform for a three variables group, e.g. $4 \cdot \mathrm{e}+$ $2 \cdot \mathrm{f}+\mathrm{g}$.

Case 2: One addition and one multiplication operations are required to perform for a two variables group, e.g. 2. a + b.

Case 3: Any addition and multiplication operation are not needed to perform for a single variables group, e.g. G2 of the Table 1 .

\section{Proposed Minimization Technique}

Any octal digit has logic adjacency with three other octal digits which are easily obtained from Karnaugh's Map which are shown in Table 2 in column name with "Adjacent Octal Pair". All minterms are arranged in ascending order. When a low weighted minterms encounter with high order adjacent minterms and it forms pair again at the time of that same higher order minterms search for pairing and form pair with 
the same lower weighted minterms as result dummy pair generate like as Quine-McCluskey's methods. As example, 0, 3, 4 and 7 this four octal minterms present. Searching for pairing initiate from 0 and when encounter with 4 , it form a pair, 0-4 again when 4 search for paring and encounter with 0 , it form a pair 4-0, which is nothing but a dummy pair.

Number of dummy pair may be increase in case of group of four adjacent minterms. Similarly for DCM, 342, 350, 374 and 382 , it impossible to apply minimization rules without converting in binary forms. If these minterms converted in BCO as $(526,536,566,576)_{8}$ we observed that both G0 and $\mathrm{G} 2$ are same digit and G2 are adjacent as shown in the Fig.1 (a). It is possible to combine four adjacent minters as $[5(2-3-6-7) 6]_{8}$ and final product term is $101 \mathrm{X} 1 \mathrm{X} 110$, as shown in Fig. 1 (b). These processes effectively reduced the number of comparisons with respected with other methods details analysis done in result and analysis sections. The observations as recorded on the below mentioned parameters during study as carried out on prototype plant are as follows:

Table 2. Adjacent pair lookup table

\begin{tabular}{|c|c|c|c|}
\hline $\begin{array}{c}\text { Octal } \\
\text { Pair }\end{array}$ & $\begin{array}{c}\text { Adjacent } \\
\text { Octal Pair }\end{array}$ & $\begin{array}{c}\text { Reduced Adjacent } \\
\text { Possible Four Pair }\end{array}$ & $\begin{array}{c}\text { Reduced } \\
\text { Adjacent } \\
\text { Pair }\end{array}$ \\
\hline 0 & $1,2,4$ & $\begin{array}{c}0-1-2-3 / 0-1-4-5 / \\
0-2-4-6\end{array}$ & $0-1 / 0-2 / 0-4$ \\
\hline 1 & $0,3,5$ & $1-3-5-7$ & $1-3 / 1-5$ \\
\hline 2 & $0,3,6$ & $2-3-6-7$ & $2-3 / 2-6$ \\
\hline 3 & $1,2,7$ & - & $3-7$ \\
\hline 4 & $0,5,6$ & $4-5-6-7$ & $4-5 / 4-6$ \\
\hline 5 & $1,4,7$ & - & $5-7$ \\
\hline 6 & $2,4,7$ & - & $6-7$ \\
\hline 7 & $3,5,6$ & - & - \\
\hline
\end{tabular}

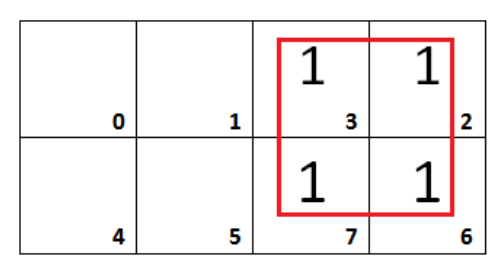

(a)

\begin{tabular}{|c|c|c|c|c|}
\hline 526 & 536 & 566 & 576 & Octal Minterms \\
\hline $\begin{array}{c}5 \\
(G 2)\end{array}$ & \multicolumn{2}{|c|}{$\begin{array}{c}2-3-6-7 \\
(G 1)\end{array}$} & $\begin{array}{c}6 \\
(\mathrm{G} 0)\end{array}$ & $\begin{array}{l}\text { Group Separations } \\
\text { and Adjacent Group } \\
\text { Formations }\end{array}$ \\
\hline 101 & \multicolumn{2}{|c|}{$\mathrm{X} 1 \mathrm{X}$} & 110 & $\begin{array}{l}\text { Binary } \\
\text { Representations }\end{array}$ \\
\hline \multicolumn{4}{|c|}{ 101X1X110 } & $\begin{array}{l}\text { Minimized Product } \\
\text { Terms }\end{array}$ \\
\hline
\end{tabular}

(b)

Fig. 1. Illustration of the proposed minimization procedure (a) Detection of adjacent minterms for G2 using Karnaugh Map, (b) Minimized product terms of $(526,536,566,576)_{8}$.

\section{A. Reduced Adjacent Table}

Any octal digit has logic adjacency with three other octal digits which are easily obtained from Karnaugh's Map which are shown in Table 2 in column name with "Adjacent Octal Pair". All minterms are arranged in ascending order. When a low weighted minterms encounter with high order adjacent minterms and it forms pair again at the time of that same higher order minterms search for pairing and form pair with the same lower weighted minterms as result dummy pair generate like as Quine-McCluskey's methods. As example, 0, 3, 4 and 7 this four octal minterms present. Searching for pairing initiate from 0 and when encounter with 4 , it form a pair, 0-4 again when 4 search for paring and encounter with 0 , it form a pair 4-0, which is nothing but a dummy pair. Number of dummy pair may be increase in case of group of four adjacent minterms. Paring is only allowed with high weighted minterms with respected to reference minterms from where searching is initiated. For avoiding this problem in software implementation, a reduced adjacency pair lookup table is prepared by omitting the repetitive pair.

Let, A0B, A2B, A4B and A5B four octal minterms. Here, $\mathrm{A}$ and $\mathrm{B}$ any octal digit which are common for all minterms. 0 , 2, 4 and 5 stores in an array and search start from next adjacent minterms are 2 and 4 so it unable to forms a pair of four only able to form pair $0-2$ and $0-4$. Next digit is 2 ; no adjacent bits are available in the higher digit. Next digit is 4 and next adjacent higher digit is 5 hence able to pair as 4-5. Final product terms are $\mathrm{A}(0-2) \mathrm{B}, \mathrm{A}(0-4) \mathrm{B}$ and $\mathrm{A}(4-5) \mathrm{B}$ i.e. $\mathrm{A} 0 \mathrm{X} 0 \mathrm{~B}, \mathrm{AX} 00 \mathrm{~B}$ and $\mathrm{A} 10 \mathrm{XB}$, by the help of prime implicants table AX00B cancel out. Here, $\mathrm{X}$ is used for those input variables having all possible logic values. This technique effectively reduces computational time with compares to other methods. Few rules are proposed here for implementing octal minterms based easy minimization technique.

\section{B. Simplification Rule 1}

For any adjacent pair of minterms, the simplified product term may be realized from the bitwise comparison between the equivalent binary form of highest minterms and lowest minterms of that pair, by simply replacing with $\mathrm{X}$ in the binary form of lowest minterms for those literals which have possessed different logic values comparing with binary form of highest minterms. This rule is also applicable for a pair of four and eight minterms.

All possible Adjacent LSP with common MSP, simplification are possible and simplified product term will contain bit pattern of MSP with simplified LSP bit pattern which are obtained by the above rule. This rule is also applicable for common LSP with adjacent MSP.

Example: In the case of two octal minterms 45 and 65, LSP are common and 4 and 6 are adjacent, and the simplified term is $1 \mathrm{X} 0$ so, $(45-65) \rightarrow(4-6) 5 \rightarrow 1 \mathrm{X} 05 \rightarrow 1 \mathrm{X} 0101$. Another example, XX14, and XX15, here bit pattern of MSP are the same and $(4-5)$ are adjacent thus the simplified term is $10 \mathrm{X}$

Sahadev Roy, "Switching Circuits Simplifications Using Binary Coded Octal Minterms," International Journal of Advanced Engineering and Management, vol. 2, no. 1, pp. 45-51, 2017. OI: http://ijoaem.org/00202-04 


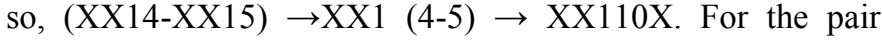
$(166,230,422,486) 10$ which equivalent in octal code is $(246$, $346,646,746) 8$ product terms to obtained binary realization of this pair required to represent 246 and 746 in binary and followed by bitwise comparison. For 2 and 7 only middle bit is matched, so final result is $\mathrm{X} 1 \mathrm{X} 100110$ which is illustrated in Fig. 2.

\begin{tabular}{|c|c|c|c|c|c|c|c|c|c|}
\hline $\begin{array}{c}\text { Octal adjacent } \\
\text { minterms }\end{array}$ & \multicolumn{9}{|c|}{$1^{\text {st }}$ and $4^{\text {th }}$ octal minterms } \\
\hline 246 & \multicolumn{3}{|c|}{ MSP } & \multicolumn{3}{|c|}{$2^{\text {nd }} L S P$} & \multicolumn{3}{|c|}{ LSP } \\
\hline 346 & \multicolumn{3}{|c|}{$2-7$} & \multicolumn{3}{|c|}{4} & \multicolumn{3}{|c|}{6} \\
\hline 646 & 0 & 1 & 0 & \multirow{2}{*}{1} & \multirow{2}{*}{0} & \multirow{2}{*}{0} & \multirow{2}{*}{1} & \multirow{2}{*}{1} & \multirow{2}{*}{0} \\
\hline 746 & 1 & 1 & 1 & & & & & & \\
\hline & $\mathrm{X}$ & 1 & $x$ & 1 & 0 & 0 & 1 & 1 & 0 \\
\hline
\end{tabular}

Fig. 2. Illustration of simplification rule 1 .

\section{Simplification Rule 2}

For any pair of adjacent LSP, the bit pattern of MSP would not match due to the difference of bit $\mathrm{X}$ with the presence of either 1 or 0 in other group pairing are allowed between them considering that $\mathrm{X}$ as 1 or 0 as per requirement. Changing of $\mathrm{X}$ into 1 or 0 allows only for a particular group contain a large number of $X$ with rest. In this case, a number of the simplified term must be two: one with the same bit pattern which contains that $\mathrm{X}$ and other is non-minimized MSP bit pattern with minimized LSP bit pattern. This rule is also applicable for the identical bit pattern of LSP.

Example: Let consider two partial minimized terms XX14 and X11-5. Here 4 and 5 logic adjacent LSP but MSP possess different bit pattern. This difference is abolished if middle MSP bit $\mathrm{X}$ is considered as 1 because $\mathrm{X}$ can be any value 0 or 1 . Here divided bit pattern of XX1-4as X01-4 and $\mathrm{X} 11-4$ to match with X11-5. So pairings are done as (X01-4, $\mathrm{X} 11-4)$ and (X11-4, X11-5). So, (X01-X11)4 $\rightarrow$ XX1-4 $\rightarrow$ $\mathrm{XX} 1100$ which is same bit pattern as initially consider. Other pairs are also minimized using rule 1 as (X11-4, X11-5) $\rightarrow$ $\mathrm{X} 11(4-5) \rightarrow \mathrm{X} 1110 \mathrm{X}$, so non-minimized bit pattern follows MSP portion and LSP with minimized bit pattern. This rule is also applicable for multiple bits change only when in a group like XX14 and 1115 and its minimized terms are XX1100 and $11110 \mathrm{X}$. Further pairing is not possible because of third MSB bit $1 \mathrm{XX} 7, \mathrm{X}$ not permitted to change 1 to match with another group.

\section{SimPLifICATION PROCEDURE}

For simplification, first group of all minterms according to ascending order of LSP while listing only remaining digits. Form subgroup considering if the number of the digit is more than one. Subgroups are needed to formed still all MSP is separated. Now consider all MSP in a group as minterms of an independent function and minimized. If any minterms cannot pair i.e. isolated minterms with other, remain unchanged keep that same. Noted all minimized bit pattern of MSP term using rule 1. Now consider group numbers (LSP) are minterms and minimized using rule 2 . In this way all prime implicants are generate.

Selection of the smallest number of prime implicants is done by using 'Prime Implicants Chart' proposed by QuineMcCluskey or 'Cover Table' proposed by S.R. Das [17]. Lastly variable assignment to each simplified product terms as 1 replaced with proper variable according to position without complemented form, 0 replaced with complemented form and $\mathrm{X}$ eliminated from the expression.

\section{A. Simplification Example 1}

Late a six variables switching function and it's minterms are given Eq. (6).

$$
\begin{array}{r}
f(A, B, C, D, E, F) \\
=\sum \mathrm{m}\left(\begin{array}{c}
0,2,7,8,10,13,16 \\
18,24,26,29,31,32,34,37 \\
39,40,42,45,47,48,50 \\
53,55,56,58,61,63
\end{array}\right)
\end{array}
$$

Using decimal coded minterms, it is hard to realize minimized product terms. Since given minterms are in decimal coded, these terms have to convert in equivalent octal number. This step is initially avoided when designer realized decimal coded minterms from truth table and convert in octal coded minterms. Conversion from binary to decimal is required more mathematical operation with comparison binary to octal conversion. Recommended for direct conversion from truth table and not follow the way binary to octal realization via decimal conversion

Equivalent octal minterms of Eq. (6) are express in Eq. (7) Here a number of MSP is eight only.

$$
f(A, B, C, D, E, F) \quad\left(\begin{array}{c}
00,02,07,10,12,15,20,22, \\
30,32,34,37,40,42,45,47 \\
50,52,55,57,60,62,65,67, \\
70,72,75,77
\end{array}\right)
$$

Groups are formed by checking the last digit of minterms presented in the equation shown in Table 3.

Table 3. Grouping according LSP

\begin{tabular}{|c|c|c|c|}
\hline Group 0 & Group 2 & Group 5 & Group 7 \\
\hline 0 & 0 & - & 0 \\
\hline 1 & 1 & 1 & - \\
\hline 2 & 2 & - & - \\
\hline 3 & 3 & 3 & 3 \\
\hline 4 & 4 & 4 & 4 \\
\hline 5 & 5 & 5 & 5 \\
\hline 6 & 6 & 6 & 6 \\
\hline 7 & 7 & 7 & 7 \\
\hline
\end{tabular}


In the case of the tabular method, we have to count all 1 present in each minterms for grouping only but in proposed method only the last digit. The proposed method effectively reduces simplification complexity. No sub-grouping is required for this example. Minimization of group 0 and group 2 is produced the same result XXX because it is contained all number from 0 to 7 . For minterms $1-3-7-5,1-5-4-6$ is an adjacent pair of four minterms. 0-4 and 3-7 are logic adjacent. To determine minimized product terms, by realizing binary from of lowest and highest minterms of the pair and change bit difference by $\mathrm{X}$ which indicates complimented literals. Minimized literal with group digit tabulated is Table 4.

Table 4. Minimization table of MSP part

\begin{tabular}{|c|c|c|c|c|}
\hline & Group 0 & Group 2 & Group 5 & Group 7 \\
\hline Octal Digit & $\begin{array}{c}0,1,2,3, \\
4,5,6,7\end{array}$ & $\begin{array}{c}0,1,2,3, \\
4,5,6,7\end{array}$ & $1,3,5,7$ & 0,4 \\
\hline $\begin{array}{c}\text { Product } \\
\text { terms }\end{array}$ & $\mathrm{XXX}$ & $\mathrm{XXX}$ & $\mathrm{XX1}$ & $\mathrm{X} 00$ \\
\hline Octal Digit & & & $4,5,6,7$ & 3,7 \\
\hline $\begin{array}{c}\text { Product } \\
\text { terms }\end{array}$ & & $1 \mathrm{XX}$ & $\mathrm{X} 11$ \\
\hline
\end{tabular}

Identification of adjacent LSP is done by the help of Table 2. Here 0-2 and 5-7 are adjacent numbers. MSP bit pattern of group 0 and 2 are same; so further simplification is possible using rule 2 and minimized product term as $\mathrm{XXX}(0-2) \rightarrow$

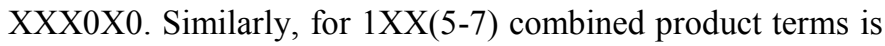
1XX1X1. XX15-X117 simplified using rule 3 and simplified product terms are XX1101, X111X1. MSP pattern X00 of group 7 not match with other, so further simplification is not possible. So simplified product terms is X00111. In this ways prime implicants are generated which are shown in Fig.3. The generated minterms are needed to test for determine essential prime implicants using prime implicants chart or using Cover Table methods. Since, proposed methods prevent to generate dummy pair less number of prime implicants are generate, here all generated prime implicants are essential prime implicants.

Variable assignments to each simplified product terms which are the prime implicants are shown in Fig. 3 are replaced with proper variable according to position without complemented form, 0 replaced with complemented form and $\mathrm{X}$ eliminate. The final minimize expression of Eq. (6) or Eq. (7) is presented as Eq. (8).

Cost and expression are same as in K-Map simplification method [18], but easier and minimum effort is required compare with other manual method of synthesis. Same minimization result will be obtained if formations of group are based on MSP.

\section{B. Simplification Example 2}

For the Eq. 3, minterms are described using octal code. Each digit of the octal representation here consider as group and arrange as increasing order of G0, G1 and G2 as Table 5. Details combinations are shown in this table. Here, (a), $\cdots$ (m) indicates first combinations. In the second combination are indicated by $(\mathrm{a}, \mathrm{d}), \cdots(\mathrm{k}, \mathrm{l})$. Only possible third combination is indicated by (c, f, k, l). By using essential prime implicates table we able to minimize easily.

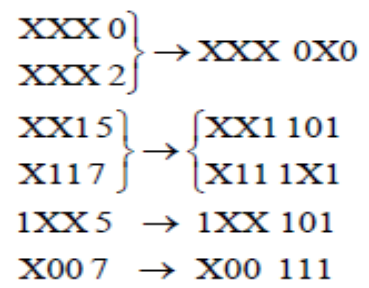

Fig. 3. Illustration of prime implicants generations.

\section{COMPARISON AND ANALYSIS}

DCM is not suitable for minimization because all simplifications approached are based on binary representation or Gray code. But binary numbers are not suitable for realization due to a number of digits. Reconversions from decimal into binary number increases unnecessary complexity in minimization of multiple inputs logic synthesis [11]. Few minimization algorithms are reported using DCM like as 'Minterms Set Method' [12], 'RAD Tree' [13]. Karnaugh map [14] is also used in manual minimization technique using DCM, but proper minimization method becomes complicated for more than four input switching system. The Karnaugh map method of minimization technique is quite simple and straight forward approach for four variable switching systems. From the case of five inputs, variable map divided into two blocks and for six input system have four blocks. In general for $\mathrm{n}$ input systems, $2^{(n-4)}$ number sub-blocks for and each subblock has sixteen cell and complicated procedure to determine adjacent cell of different blocks. Another drawback of this procedure is that numbers of cells are $2 n$ though the numbers of minterms may be less. Karnaugh map is manual minimization methods and software implementation is quite tricky. In proposed method number of the group depends on presented minterms. Maximum elements on each group are seven and it is quite easy to determine logic adjacency using Table 2.

The Quine-McCluskey algorithm is basically NP-hard type minimization technique and number of comparison increase with the number of input variables which increase the runtime of this algorithm. Propose minimization method required compression is less than the Quine-McCluskey algorithm which reduces runtime effectively.

In Quine-McCluskey's method, simplified product term for four pairs is obtained by minimum three comparisons, but using the rule 1 , by only single comparison simplified product term is obtained easily. This technique effectively reduces complexity and also improves the speed of simplification. $33 \%$ less operations shown in Fig. 4. 
Table 5. Octal minterms combination tables

\begin{tabular}{|c|c|c|c|c|c|c|c|}
\hline $\begin{array}{c}\text { Octal } \\
\text { Minterms }\end{array}$ & $\mathrm{G} 2$ & G1 & G0 & Combinations & First Combination & Second Combination & Third Combination \\
\hline 002 & $\mathbf{0}$ & $\mathbf{0}$ & 2 & $0(0,1) 2$ & $000 \times 2$ & \multirow{16}{*}{$\begin{array}{l}(\mathrm{a}, \mathrm{d}) 00 \mathrm{XX} 2 \mathrm{I} 2 \\
(\mathrm{c}, \mathrm{b}) 00 \mathrm{XX} 2 \mathrm{I} 2 \\
(\mathrm{c}, \mathrm{f}) \mathrm{X} 0 \mathrm{XX12}\end{array}$} & \multirow{16}{*}{$(\mathrm{c}, \mathrm{f}, \mathrm{k}, \mathrm{l}) \times \mathrm{X}$ X1 X10 I4 } \\
\hline 012 & $\mathbf{0}$ & 1 & 2 & $0(0,2) 2$ & (0)X0 2 (b) & & \\
\hline 022 & $\mathbf{0}$ & 2 & 2 & $0(1,3) 2$ & $00 \times 12 \quad$ (c) & & \\
\hline 032 & $\mathbf{0}$ & 3 & 2 & $0(2,3) 2$ & $001 \times 2 \quad$ (d) & & \\
\hline 042 & $\mathbf{0}$ & 4 & 2 & $0(0,4) 2$ & 0 X00 2 & & \\
\hline 112 & 1 & 1 & 2 & \multirow{2}{*}{$1(1,3) 2$} & \multirow{2}{*}{$10 X 12$} & & \\
\hline 132 & 1 & 3 & 2 & & & & \\
\hline 013 & $\mathbf{0}$ & 1 & 3 & \multirow{4}{*}{$\begin{array}{l}0(1,3) 3 \\
0(1,5) 3 \\
0(3,7) 3 \\
0(5,7) 3\end{array}$} & \multirow{4}{*}{$\begin{array}{llll}\mathbf{0} & 0 X 1 & 3 & (\mathrm{~g}) \\
\mathbf{0} & X 01 & 3 & (\mathrm{~h}) \\
\mathbf{0} & X 11 & 3 & (\mathrm{i}) \\
\mathbf{0} & 1 \mathrm{X} 1 & \mathbf{3} & (\mathrm{j})\end{array}$} & & \\
\hline 033 & $\mathbf{0}$ & 3 & 3 & & & & \\
\hline 053 & $\mathbf{0}$ & 5 & 3 & & & & \\
\hline 073 & $\mathbf{0}$ & 7 & 3 & & & & \\
\hline 016 & $\mathbf{0}$ & 1 & 6 & \multirow{2}{*}{$0(1,3) 6$} & \multirow{2}{*}{$00 \times 16 \quad(k)$} & & \\
\hline 036 & $\mathbf{0}$ & 3 & 6 & & & & \\
\hline 116 & 1 & 1 & 6 & \multirow[t]{3}{*}{$1(1,3) 6$} & \multirow[t]{2}{*}{$10 \times 16$} & & \\
\hline 136 & 1 & 3 & 6 & & & & \\
\hline 156 & 1 & 5 & 6 & & $1 \mathrm{X} 016 \quad(\mathrm{~m}) \quad \mathrm{I} 1$ & & \\
\hline
\end{tabular}

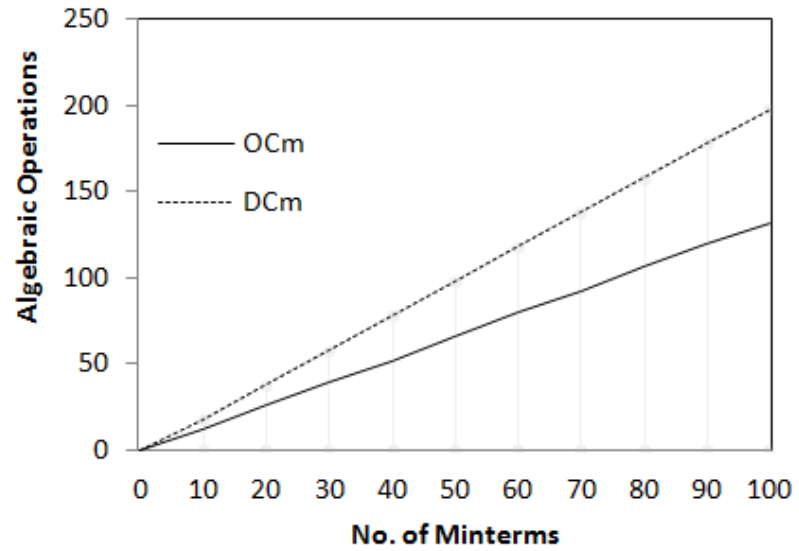

Fig. 4. Required operations between Octal Coded Minterms and Decimal Coded Minterms

Another advantage to use reduced adjacent pair table, it is not required to search for pair in lower weighted minterms with respected to reference bit, because it already search done when those lowest weighted bits acts as reference bit. This method prevents to produce dummy pairs and dummy prime implicants.

\section{CONCLUSIONS}

Here we proposed a new approach for simplification of switching circuit based on the octal representation of minterms. Some concluding observations from the investigation are given below. Decimal coded minterms are not useful in minimization process except representations of input conditions. For minimization all decimal minterms require to reconvert in binary form no direct implementation methods not present, these difficulties is successfully overcome using proposed octal minimization technique. Minimal solution is a legitimate logic implementation with a minimal number of input variables which reduces complexity effectively for gate level implementation by using octal coded minterms. The proposed method is required less number of comparisons. This method can easily be used for any number of variables.

\section{REFERENCES}

[1] S. Roy and C.T. Bhunia, "On Synthesis of Combinational Logic Circuits," International Journal of Computer Applications, vol.127, no.1, pp.21-26, 2015. https://doi.org/10.5120/ijca2015906311

[2] S. Roy and C.T. Bhunia, 2014, "Minimization Algorithm for Multiple Input to Two Input Variables," Proc. Int. IEEE Conf. on Control, Instrumentation Energy and Communication, CIEC14, pp. 555-557. https://doi.org/10.1109/ciec.2014.6959150

[3] S. Roy and C.T. Bhunia, "Constraints Analysis for Minimization of Multiple Inputs Logic Programming," Proc. of Int. Elsevier Conf. on Signal and Speech Processing, ICSSP-14, pp. 61-64, 2014.

[4] C.E. Shannon, "A Symbolic Analysis of Relay and Switching Circuits," Trans. of the American Institute of Electrical Engineers, vol.57, no.12, pp.713-723, 1938. https://doi.org/10.1109/ee.1938.6431064

[5] E.W. Veitch, "A Chart Method for Simplifying Truth Functions," Proc. of the 1952 ACM national meeting, pp.127-133, 1952. https://doi.org/10.1145/609784.609801

[6] M. Karnaugh, "The Map Method For Synthesis of Combinational Logic Circuits," Trans. of the Communication and Electronics, American Inst. of 
Electrical Engineers, Part I, vol.72, no.5, pp.593-599,

[7] W. V. Quine, "The Problem of Simplifying Truth Functions," Amer. Math. Monthly, vol.59, no.8, pp. 521531, 1952. https://doi.org/10.2307/2308219

[8] S. Roy, "An Efficient Technique For Switching Functions Simplification," International Journal of Advanced Engineering and Management, vol. 2, no. 1, pp. 21-28, 2017. https://ijoaem.org/00201-9

[9] S.J. Hong, R.G. Cain and D.L. Ostapko, "MINI: A Heuristic Approach for Logic Minimization," IBM J. of Res. \& Dev., pp.443-458, 1974. https://doi.org/10.1147/rd.185.0443

[10] J. Hlavicka and P. Fišer, “ BOOM - A Heuristic Boolean Minimizer," Computers and Informatics, vol. 22, no. 1, pp. 19-51,

2003. https://doi.org/10.1109/iccad.2001.968667

[11] K. Chakrabarty and S.R. Das, "Test-Set Embedding Based on Width Compression for Mixed-Mode BIST," IEEE Trans. on Instrumentation and Measurement, vol. 49, no. 3, pp. 671-678, 2000. https://doi.org/10.1109/19.850413

[12] J. Roh, "Virtual Ground Monitoring for High Fault Coverage of Linear Analog Circuits," J. of Semiconductor Technology and Science, vol.2 no.3 pp.226-232, 2002.

[13] R. Saha, V. Nandi, S. Roy and C.T. Bhunia, "Design and Verifications of Efficient Arbiter of SoC's On-Chip Bus," Proc. 3rd Int. IEEE Conference on Electronics and Communication Systems (ICECS), Paper No. 685, 2016. 1953.https://doi.org/10.1109/tce.1953.6371932

[14] S. Roy, R. Saha and C.T. Bhunia, "Multiple Inputs Combinational Logic Minimization by Minterms Set," Proceedings of the International Conference on Recent Cognizance in Wireless Communication \& Image Processing, pp. 133-140, 2016. https://doi.org/10.1007/978-81-322-2638-3_15

[15] Y. Omura, "A New Basic Element for Neural Logic Functions and Capability in Circuit Applications," J. of Semiconductor Technology and Science, vol. 2 no.1, pp.70-81, 2002. https://doi.org/10.1002/9781118487914.ch17

[16] R. Serrell, "Elements of Boolean Algebra for the Study of Information-Handling Systems." Proceedings of the IRE, vol.41, no.10, pp.1366-1380, 1953. https://doi.org/10.1109/jrproc.1953.274313

[17] S. R. Das, "An approach for simplifying switching functions by utilizing the cover table representation," IEEE Transactions on Computers, vol. 3, pp. 355-359, 1971. https://doi.org/10.1109/tc.1971.223244

[18] S. Roy, "Breakup Algorithm for Switching Circuit Simplifications," International Journal of Advanced Engineering and Management, vol. 1, no. 1, pp.1-11, 2016. https://ijoaem.org/001-2 